\title{
Eruptive Lentigines after Adalimumab Therapy
}

\author{
Efstathia Pasmatzi*, Alexandra Monastirli, Sophia Georgiou, Dionysios Tsambaos \\ Department of Dermatology, School of Medicine, University of Patras, Patras, Greece \\ Email: "pasmatzi@med.upatras.gr
}

Received 22 February 2016; accepted 18 April 2016; published 21 April 2016

Copyright (C) 2016 by authors and Scientific Research Publishing Inc.

This work is licensed under the Creative Commons Attribution International License (CC BY). http://creativecommons.org/licenses/by/4.0/

c) (i) Open Access

\begin{abstract}
Adalimumab, a TNF-alpha antagonist, is the first fully humanized recombinant immunoglobulin G1 (IgG1) monoclonal antibody. It is presently widely used in the systemic treatment of rheumatoid arthritis, inflammatory bowel disease, moderate and severe psoriasis and hidradenitis suppurativa. However, its administration is associated with a two-fold risk of severe and possibly fatal infections and in some rare cases with congestive heart failure, lymphoma, lupus-like syndrome, cytopenias, hepatotoxicity and development of demyelinating neurological disorders. Furthermore, the occurrence of various types of melanocytic skin lesions has been reported during treatment with adalimumab. In the present paper we report the case of a female psoriatic patient who developed eruptive lentigines following treatment with this compound.
\end{abstract}

\section{Keywords}

\section{Lentigines, Melanocytes, TNF- $\alpha$, Adalimumab, Psoriasis}

\section{Introduction}

Emerging biologic agents target specific key mediators in the immunopathogenesis of various immune and inflammatory diseases [1] and presently include three distinct classes of compounds: The inhibitors of interleukin-12 (IL-12)/interleukin-23 (IL-23), interleukin-17 (IL-17) and the inhibitors of tumor necrosis factor-alpha (TNF-alpha). The members of the latter class (etanercept, infliximab, adalimumab) are widely used in the systemic treatment of various autoimmune and inflammatory diseases, including moderate to severe plaque psoriasis. Recently, the occurrence of various types of melanocytic lesions has been reported during treatment with TNF-alpha inhibitors [2]-[4]. In the present paper we report the case of a psoriatic female patient who developed eruptive lentigines, subsequent to treatment with adalimumab.

*Corresponding author. 


\section{Case Report}

In January 2010 the dermatologist of a 75-year-old woman with a 35-year history of chronic plaque psoriasis with arthropathy initiated a subcutaneous treatment with 40 mg adalimumab (Humira, Abbott Laboratories Ltd, Athens, Greece) every second week. Pre-treatment assessment for tuberculosis with tuberculin test was negative and chest radiograph was normal.

In February 2013, after three years of continuous treatment with adalimumab, she was admitted to the Department of Internal Medicine with a tuberculous peritonitis which was successfully treated with rifampicin, isoniazid and ethambutol (to be reported elsewhere).

Because of a relapse of her chronic plaque psoriasis due to the discontinuation of adalimumab, the patient (Fitzpatrick skin type IV) presented to the Department of Dermatology, University of Patras, Greece in September 2013. Apart from the psoriatic lesions, physical investigation revealed the presence of numerous and disseminated asymptomatic brown macular lesions (Figure 1), that developed in a short span of time 2 months after onset of adalimumab therapy, on both the apparently normal skin and the resolving psoriatic plaques. Her family history for lentigines and melanoma was negative.

She had previously been treated by her home town dermatologists with different topical (corticosteroids, dithranol, emollients) and systemic regimens (cyclosporine, acitretin, leflunomide). However, she had received no phototherapy or photochemotherapy. Her past medical history was remarkable for coronary heart disease, paroxysmal atrial fibrillation and cataract.

Routine laboratory tests including a complete blood count, blood chemistry, urinalysis, immunological and serological investigations (tests for syphilis, HSV 1 \& 2, HIV 1 \& 2, hepatitis A, B and C and CMV) were performed. Their results were either negative or within normal limits and chest X-ray and electrocardiogram were unremarkable. An informed consent was obtained from her to perform the biopsies, take photographs and report her case.

Histological examination of the brown macular lesions obtained from various sites showed distinct prolongation of rete ridges and increased numbers of uniformly dispersed melanocytes in the basal layer with variable hyperpigmentation (Figure 2). Occasionaly, a sparse lymphohistiocyticinfiltrate and considerable numbers of melanophages could be seen in the papillary dermis. Thus, the diagnosis of eruptive lentigines was established. Biopsy specimens of adjacent clinically unaffected skin were essentially normal (Figure 3). The patient was instructed to topically apply anthralin ointment $2 \%$ once daily (short contact therapy) on her psoriatic plaques. However, she was lost to follow-up.

\section{Discussion}

TNF-alpha is a cytokine known to play a significant role in the surveillance of infectious and neoplastic disorders [3]. Adalimumab, a TNF-alpha antagonist, is the first fully humanized recombinant immunoglobulin G1

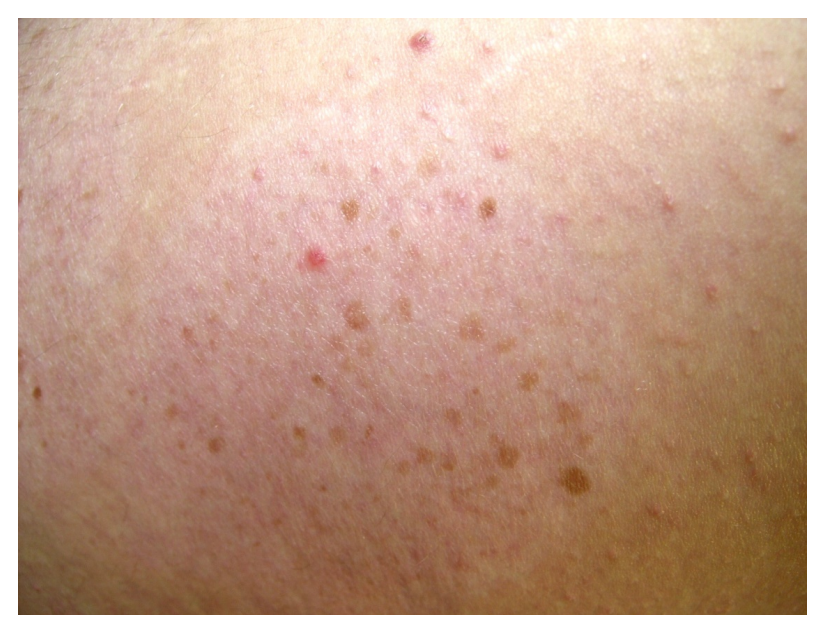

Figure 1. Eruptive lentigines in the resolving psoriatic plaques of a patient treated with adalimumab. 


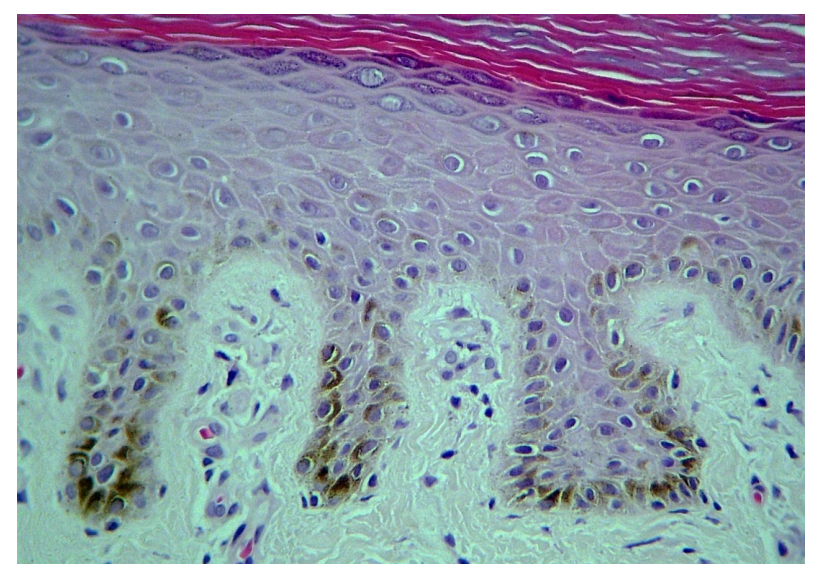

Figure 2. Distinct prolongation of rete ridges and increased numbers of melanocytes in the basal layer $(\times 400$; Hematoxylin-eosin).

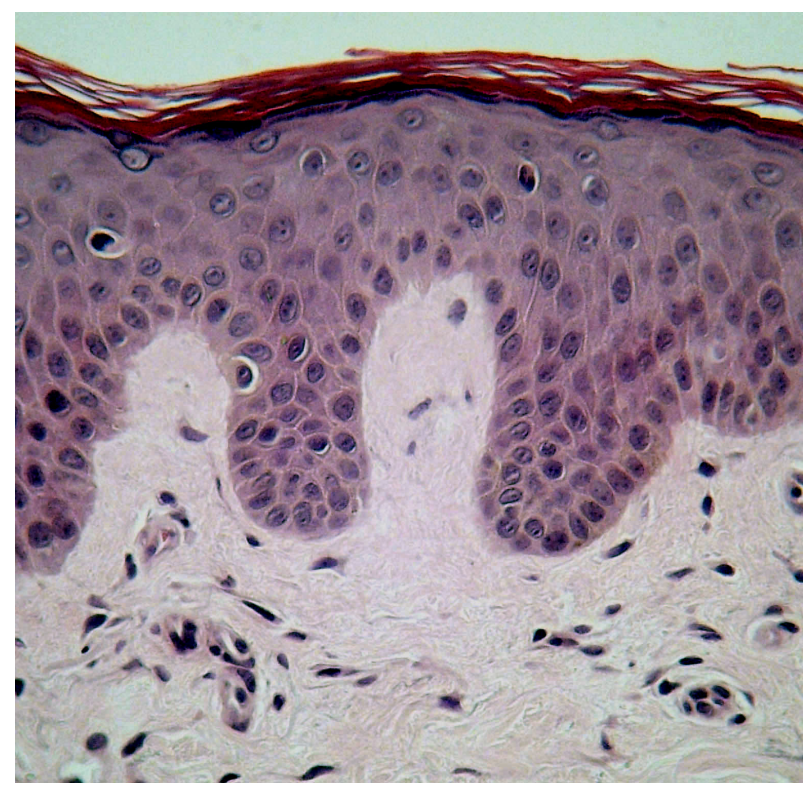

Figure 3. Histological aspects of adjacent clinically unaffected skin of a patient treated with adalimumab $(\times 400$; Hematoxylin-eosin).

(IgG1) monoclonal antibody. It exerts its inhibitory effects on TNF-alpha by occupying the corresponding binding site and competitively inhibiting the binding of this cytokine to its receptor (TNFR) [3] [5].

Adalimumab is presently widely used in the systemic treatment of rheumatoid arthritis, inflammatory bowel disease, moderate and severe psoriasis and hidradenitis suppurativa. However, its administration is associated with a two-fold risk of severe and possibly fatal infections (deep fungal infections, bacterial infections including reactivation of tuberculosis, atypical mycobacterial infections, parasitic infections) [6]. Furthermore, serious but relatively rare side effects of this compound include congestive heart failure, lymphoma (particularly hepatosplenic T-cell lymphoma), lupus-like syndrome, cytopenias, hepatotoxicity and development of demyelinating neurological disorders [6].

Cutaneous side effects of adalimumab include reactions at the injection site, lupus erythematosus, urticaria, pustular dermatoses, leukocytoclastic vasculitis, non-specific rashes and flare of psoriasis [7]. In some rare cases, the use of this compound has been reportedly associated with the occurrence of various types of melanocytic skin lesions, such as melanoma, nevi, hyperpigmentation and lentigines, with the latter representing the simplest 
form of them.

Rapid development of hyperpigmented skin lesions is reportedly associated with chemotherapeutic agents and bullous disorders [8]. Phototherapy and photochemotherapy, but also topical corticosteroids and vitamin D derivatives are known to induce the occurrence of lentigines in treated psoriatic patients [9]. Our patient has never received phototherapy, photochemotherapy or topical vitamin $\mathrm{D}$ derivatives. About two decades prior to her presentation to the Department of Dermatology she had been treated with topical steroids; it seems very unlikely, however, that the occurrence of eruptive lentigines may be attributed to the latter.

To the best of our knowledge, the patient presented here is the second case who developed eruptive lentigines subsequent to adalimumab therapy. In both, the case reported by Santos-Juanes et al. (2008) [10] and in our case, the development of these lesions started two months after the onset of treatment. This chronological association between adalimumab administration and the development of eruptive lentigines suggests that this drug may be implicated in the etiopathogenetic mechanisms of the latter. Since an intact immune system normally inhibits the proliferation of melanocytic lesions [11], it seems likely that the immunossupression caused by adalimumab either leads to a dysregulation of melanocytic stem cell division [12] or facilitates the rapid and possibly uncontrolled proliferation of melanocytes in genetically predisposed patients [13] [14].

Furthermore, sincethe induction of cutaneousbenign and malignantmelanocytic proliferation seems to be a property shared by adalimumab and all other biologic agents, in patients who are treated with these compounds a close monitoring during treatment and a rigorous follow-up are highly recommended.

\section{Conclusion}

The results of the present paper taken together with those reported by other groups, clearly indicate that adalimumab and all other biologic agents, apart from their significant and serious side effects, are capable of inducing the cutaneous benign and malignant melanocytic proliferation. Thus, a close monitoring during treatment and a rigorous follow-up of patients treated with these compounds, are of essential importance.

\section{References}

[1] Gaspari, A.A. and Tyring, S. (2015) New and Emerging Biologic Therapies for Moderate-to-Severe Plaque Psoriasis: Mechanistic Rationales and Recent Clinical Data for IL-17 and IL-23 Inhibitors. Dermatologic Therapy, 28, 179-193. http://dx.doi.org/10.1111/dth.12251

[2] Bardazzi, F., Magnano, M., Antonucci, V.A., Balestri, R., Sgubbi, P. and Patrizi, A. (2012) Lentigines in Previous Psoriatic Plaques in a Patient Treated with Infliximab. European Journal of Dermatology, 22, 698-699.

[3] Kouklakis, G., Efremidou, E., Pitiakoudis, M., Liratzopoulos, N. and Polychronidis, A. (2013) Development of Primary Melanoma during Treatment with a TNF- $\alpha$ Antagonist for Severe Chron's Disease: A Case Report and Review of the Hypothetical Association between TNF- $\alpha$ Blockers and Cancer. Drug Design, Development and Therapy, 7, 195199.

[4] Sousa, M., Freitas, J.P., Antunes, J., Soares-de-Almeida, L. and Filipe, P.L. (2014) Appearance of de Novo Dysplastic Spitzoid Compound Naevus in an Adalimumab-Treated Psoriatic Patient: Case Report and Review of the Possible Causal Relationship with TNF- $\alpha$ Blockers. Australasian Journal of Dermatology, 55, 156-157. http://dx.doi.org/10.1111/ajd.12170

[5] Hu, S., Liang, S., Guo, H., Zhang, D., Li, H., Wang, X., Yang, W., Qian, W., Hou, S., Wang, H., Guo, Y. and Lou, Z. (2013) Comparison of the Inhibition Mechanisms of Adalimumab and Infliximab in Treating Tumor Necrosis Factor $\alpha$-Associated Diseases from a Molecular View. Journal of Biological Chemistry, 288, 27059-27067. http://dx.doi.org/10.1074/jbc.M113.491530

[6] Scheinfeld, N. (2005) Adalimumab: A Review of Side Effects. Expert Opinion on Drug Safety, 4, 637-641. http://dx.doi.org/10.1517/14740338.4.4.637

[7] Blomberg, M., Zachariae, C. and Granhuj, F. (2009) Hyperpigmentation of the Face Following Adalimumab Treatment. Acta Dermato-Venereologica, 89, 546-547. http://dx.doi.org/10.2340/00015555-0697

[8] Bovenschen, H.J., Tjioe, M., Vermaat, H., de Hoop, D., Witteman, B.M., Janssens, R.W., Stoof, T.J. and van de Kerkhof, P.C. (2006) Induction of Eruptive Benign Melanocytic Naevi by Immune Suppressive Agents, including Biologicals. British Journal of Dermatology, 154, 880-884. http://dx.doi.org/10.1111/j.1365-2133.2006.07189.x

[9] Rogers, M. (1995) Multiple Lentigines Confined to a Resolving Psoriatic Plaque Treated without Phototherapy. Clinical and Experimental Dermatology, 20, 446. http://dx.doi.org/10.1111/j.1365-2230.1995.tb01372.x

[10] Santos-Juanes, J., Coto, P., Mallo, S., Galacher, C., Sanchez del Rio, J. and Torre, J.C. (2008) Multiple Lentigines 
Confined to Resolving Psoriatic Plaques in a Patient Treated with Adalimumab. Dermatology, 216, 279. http://dx.doi.org/10.1159/000113947

[11] Wolf, R., Lipozencic, J., Segal, Z. and Davidovici, B. (2008) Eruptive Acrallentigines-A New Paraneoplastic Sign? Acta Dermato-Venereologica, 16, 130-132.

[12] Zattra, E., Fortina, A., Bordignon, M., Piaserico, S. and Alaibac, M. (2009) Immunosuppression and Melanocyte Proliferation. Melanoma Research, 19, 63-68. http://dx.doi.org/10.1097/CMR.0b013e328322fc20

[13] Alaibac, M., Piaserico, S., Rossi, C.R., Foletto, M., Zaccharelo, G., Carli, P. and Belloni-Fortina, M. (2003) Eruptive Melanocytic Nevi in Patients with Renal Allografts: Report of 10 Cases with Dermoscopic Findings. Journal of the American Academy of Dermatology, 49, 1020-1022. http://dx.doi.org/10.1016/S0190-9622(03)02482-4

[14] Kong, H., Sibaud, V., Turner, M., Fojo, T., Hornyak, T. and Chevreau, C. (2008) Sorafenib-Induced Eruptive Melanocytic Lesions. Archives of Dermatology, 144, 820-822. http://dx.doi.org/10.1001/archderm.144.6.820 